\title{
Asymmetric Conjugate Addition of Alkynylboronates to Enones: Rationale for the Intriguing Catalysis Exerted by Binaphthols
}

\author{
Silvina C. Pellegrinet ${ }^{*}{ }^{\dagger}$ and Jonathan M. Goodman*,‡ \\ †Instituto de Química Orgánica y de Síntesis (CONICET), Facultad de Ciencias Bioquímicas y Farmacéuticas, \\ Universidad Nacional de Rosario, Suipacha 531, Rosario (2000), Argentina \\ ${ }^{\ddagger}$ Unilever Centre for Molecular Science Informatics, Department of Chemistry, University of Cambridge, Lensfield \\ Road, Cambridge CB2 1EW, U. K.
}

\section{Supplementary Information}

List of contents:

- B3LYP/lacvp* optimized geometries of all the transition structures studied in the paper (Figures S1 and S2). Pages S2-S3.

- Selected distances (in $\AA$ ) and bond orders for all the transition structures studied in the paper and activation energies of the corresponding reactions (Tables S1 and S2). Page S4.

- B3LYP/lacvp* reaction coordinates for the conjugate additions of alkynylboronates 2a and 5a to enone 1a (Schemes S1 and S2) and discussion. Pages S5-S6.

- B3LYP/lacvp* optimized geometries of the complex between enone 1a and alkynylboronate 5a (Figures S3). Page S6.

- Cartesian coordinates and absolute energies (in hartrees), including ZPE, and number of imaginary frequencies of all stationary points reported in the paper; values of imaginary frequencies of all transition structures. Pages S7S13.

- B3LYP/lacvp* geometries and absolute energies (in hartrees), including ZPE (free energies in parentheses) of the conformers located for alkynylboronate 2a and its transition structures (Figures S4-S6). Page S14-S15.

- Energies and Atomic Coefficients of the Frontier Molecular Orbitals of the Reactants (Table S3). Page S15.

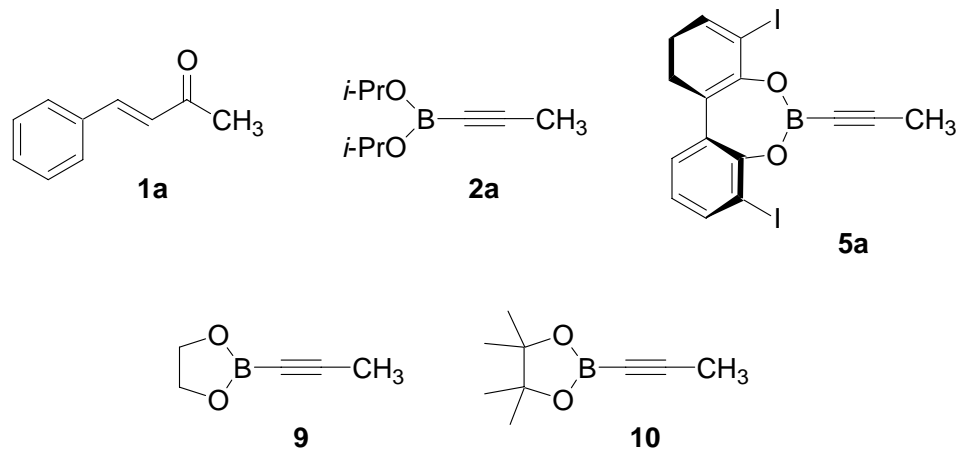



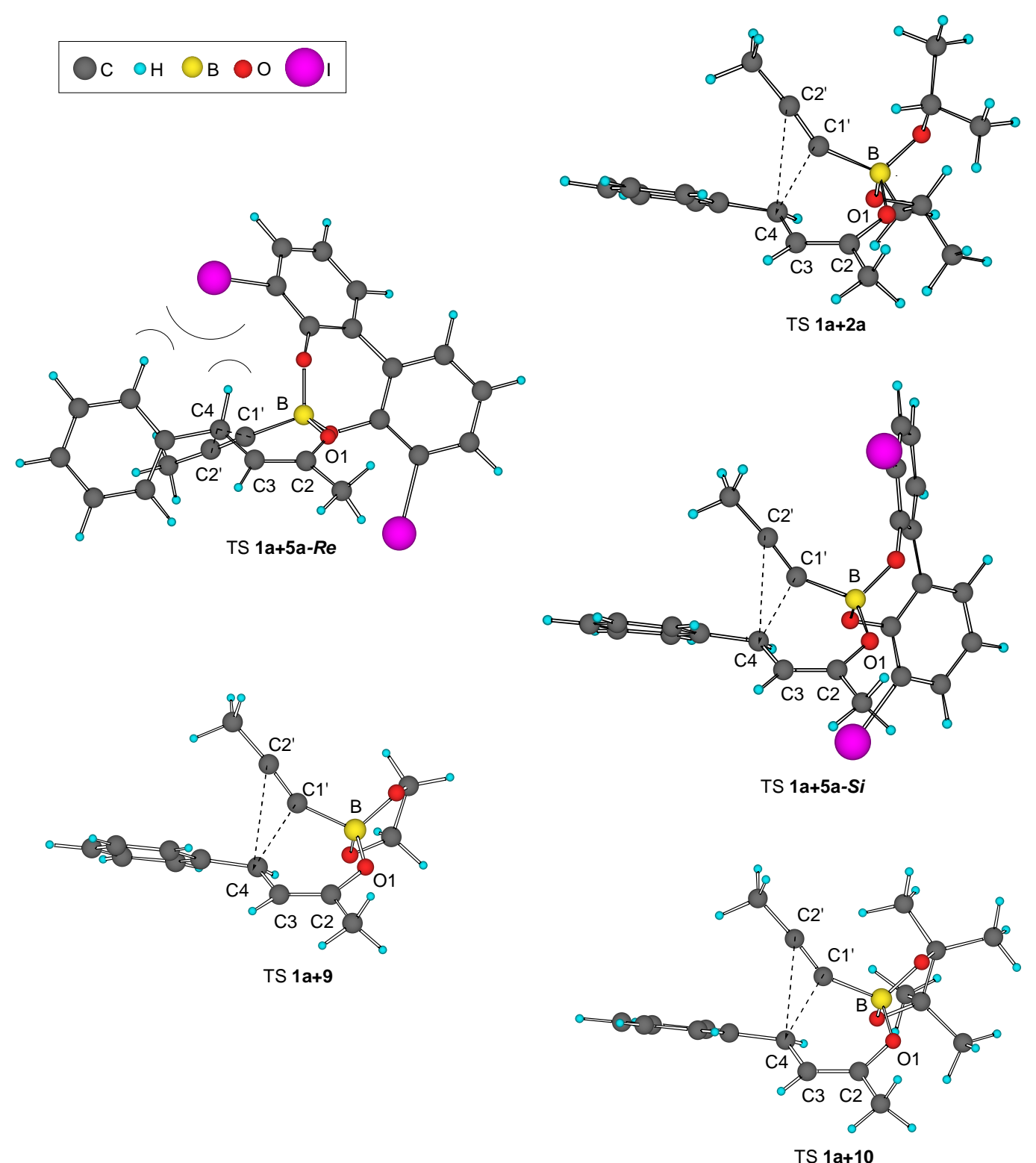

Figure S1. B3LYP/lacvp* optimized geometries of the transition structures of the conjugate additions of alkynylboronates 2a, 5a, 9 and 10 to enone 1 . 

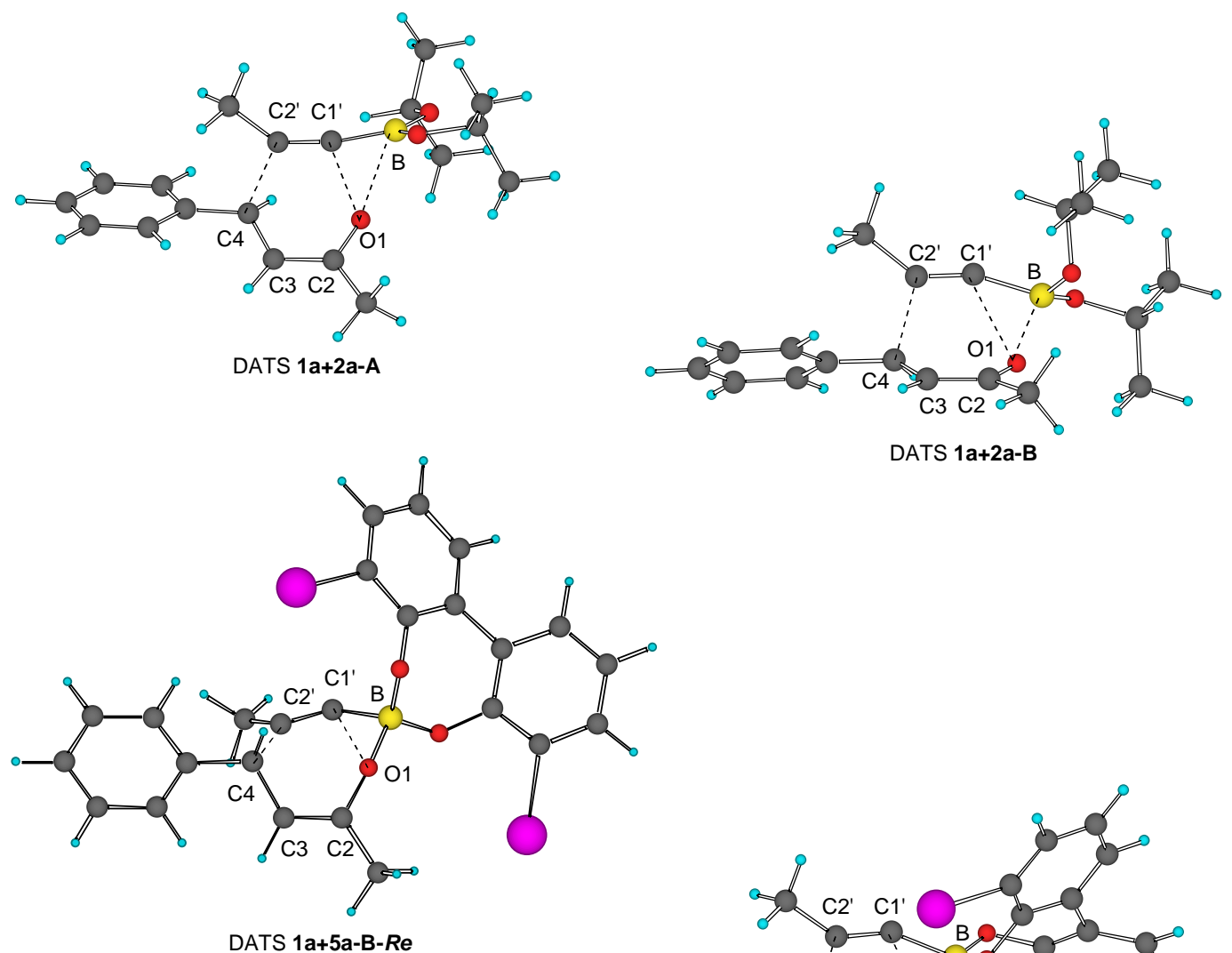

DATS 1a+2a-B

DATS 1a+5a-B-Re

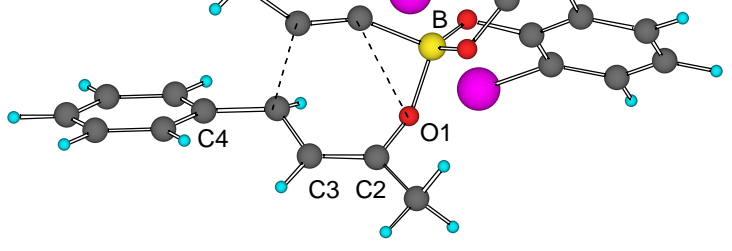

DATS 1a+5a-B-Si

Figure S2. B3LYP/lacvp* optimized geometries of the transition structures of the hetero Diels-Alder reactions of enone 1a with alkynylboronates $\mathbf{2 a}$ and $\mathbf{5 a}$. 
Table S1. Selected distances (in $\AA$, NBOs in parentheses) and B3LYP/lacvp* Activation Energies including Zero-point Energy (ZPE) Corrections, Activation Enthalpies and Activation Free Energies (in kcal mol ${ }^{-1}$, relative values in parentheses) for the TSs of the Conjugate Addition of Alkynylboronates 2a, 5a, 9 and 10 to Enone 1a

\begin{tabular}{ccccccc}
\hline $\begin{array}{c}\mathrm{TS} \\
\mathbf{1 a}+\end{array}$ & $\mathrm{B}-\mathrm{O} 1$ & $\mathrm{C} 1^{\prime}-\mathrm{C} 4$ & $\mathrm{C} 2^{\prime}-\mathrm{C} 4$ & $\begin{array}{c}\Delta \mathrm{E}^{\ddagger} \\
\left(\mathrm{kcal} \mathrm{mol}^{-1}\right)\end{array}$ & $\begin{array}{c}\Delta \mathrm{S}^{\ddagger} \\
\left(\mathrm{kcal} \mathrm{mol}^{-1}\right)\end{array}$ & $\begin{array}{c}\Delta \mathrm{G}^{\ddagger} \\
\left(\mathrm{kcal} \mathrm{mol}^{-1}\right)\end{array}$ \\
\hline $\mathbf{2 a}$ & 1.548 & 1.919 & 2.650 & 26.06 & 25.82 & 41.35 \\
& $(0.56)$ & $(0.49)$ & $(0.09)$ & $(16.32)$ & $(16.43)$ & $(14.95)$ \\
$\mathbf{5 a - R e}$ & 1.492 & 1.890 & 2.610 & 10.92 & 10.61 & 27.40 \\
& $(0.63)$ & $(0.52)$ & $(0.10)$ & $(1.18)$ & $(1.22)$ & $(1.00)$ \\
$\mathbf{5 a - S i}$ & 1.483 & 1.904 & 2.626 & 9.74 & 9.39 & 26.40 \\
& $(0.63)$ & $(0.50)$ & $(0.10)$ & $(0.00)$ & $(0.00)$ & $(0.00)$ \\
$\mathbf{9}$ & 1.505 & 1.938 & 2.659 & 23.28 & 22.84 & 38.87 \\
& $(0.60)$ & $(0.48)$ & $(0.09)$ & $(13.54)$ & $(13.45)$ & $(12.47)$ \\
$\mathbf{1 0}$ & 1.514 & 1.932 & 2.664 & 23.99 & 23.73 & 39.68 \\
& $(0.59)$ & $(0.48)$ & $(0.09)$ & $(14.25)$ & $(14.34)$ & $(13.28)$ \\
\hline
\end{tabular}

Table S2. Selected distances (in Å, NBOs in parentheses) and B3LYP/lacvp* Activation Energies including Zero-point Energy (ZPE) Corrections, Activation Enthalpies and Activation Free Energies (in kcal mol ${ }^{-1}$, relative values in parentheses) for the Transition Structures of the Hetero Diels-Alder Reactions of Enone 1a with Alkynylboronates 2a and 5a

\begin{tabular}{ccccccc}
\hline $\begin{array}{c}\mathrm{DATS} \\
\mathbf{1}+\end{array}$ & $\mathrm{B}-\mathrm{O} 1$ & $\mathrm{C} 1{ }^{\prime}-\mathrm{O} 1$ & $\mathrm{C} 2{ }^{\prime}-\mathrm{C} 4$ & $\begin{array}{c}\Delta \mathrm{E}^{\ddagger} \\
\left(\mathrm{kcal} \mathrm{mol}^{-1}\right)\end{array}$ & $\begin{array}{c}\Delta \mathrm{S}^{\ddagger} \\
\left(\mathrm{kcal} \mathrm{mol}^{-1}\right)\end{array}$ & $\begin{array}{c}\Delta \mathrm{G}^{\ddagger} \\
\left(\mathrm{kcal} \mathrm{mol}^{-1}\right)\end{array}$ \\
\hline 2a-A & 2.435 & 2.111 & 2.012 & 31.43 & 31.29 & 46.54 \\
& $(0.06)$ & $(0.30)$ & $(0.48)$ & $(11.28)$ & $(11.72)$ & $(9.50)$ \\
2a-B & 1.641 & 2.238 & 1.947 & 37.45 & 36.97 & 53.45 \\
& $(0.46)$ & $(0.20)$ & $(0.53)$ & $(17.30)$ & $(17.40)$ & $(16.41)$ \\
5a-B-Re & 1.497 & 2.371 & 1.794 & 22.36 & 21.81 & 39.37 \\
& $(0.62)$ & $(0.13)$ & $(0.64)$ & $(2.21)$ & $(2.24)$ & $(2.33)$ \\
5a-B-Si & 1.531 & 2.273 & 1.899 & 20.15 & 19.57 & 37.04 \\
& $(0.57)$ & $(0.18)$ & $(0.57)$ & $(0.00)$ & $(0.00)$ & $(0.00)$ \\
\hline
\end{tabular}




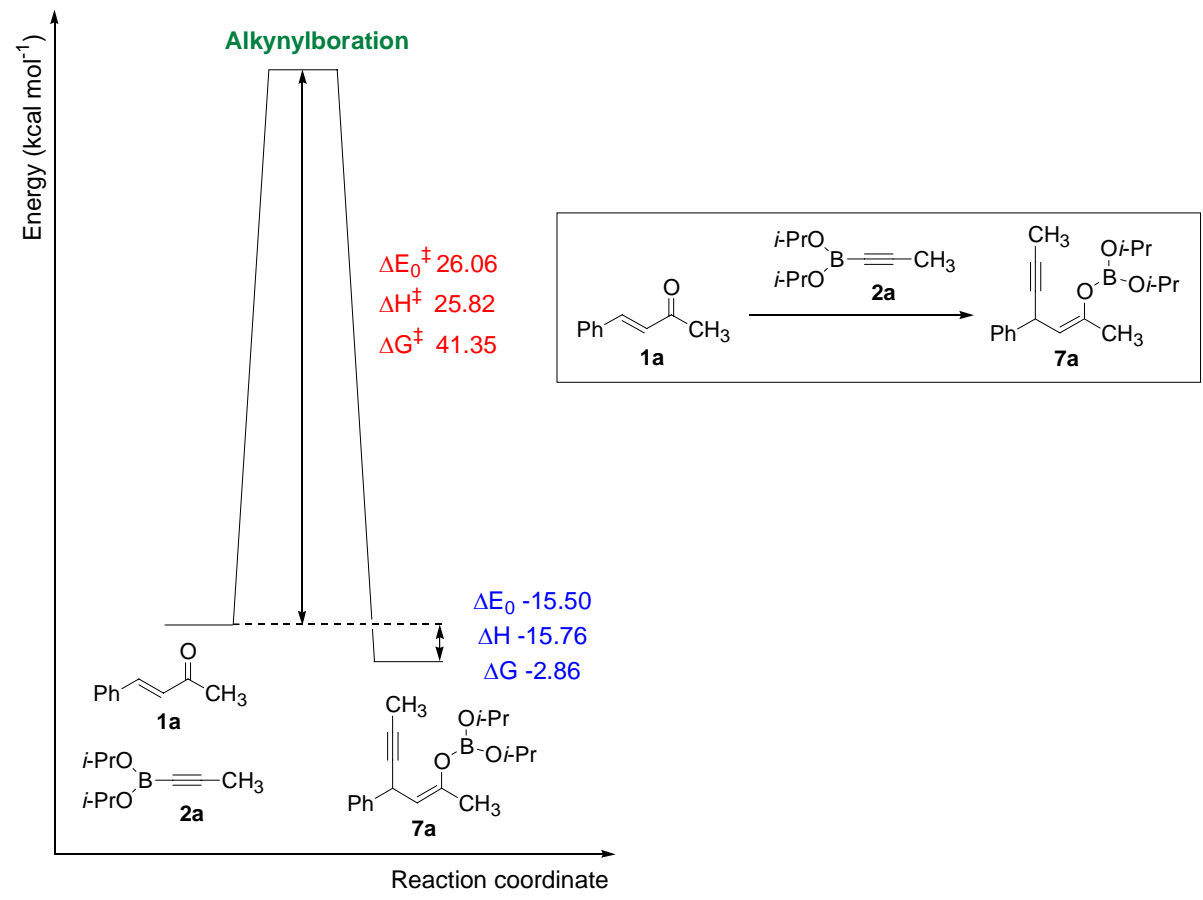

Scheme S1. B3LYP/lacvp* reaction coordinate of the conjugate addition of alkynylboronate 2a to enone 1a. All values in kcal $\mathrm{mol}^{-1}$.

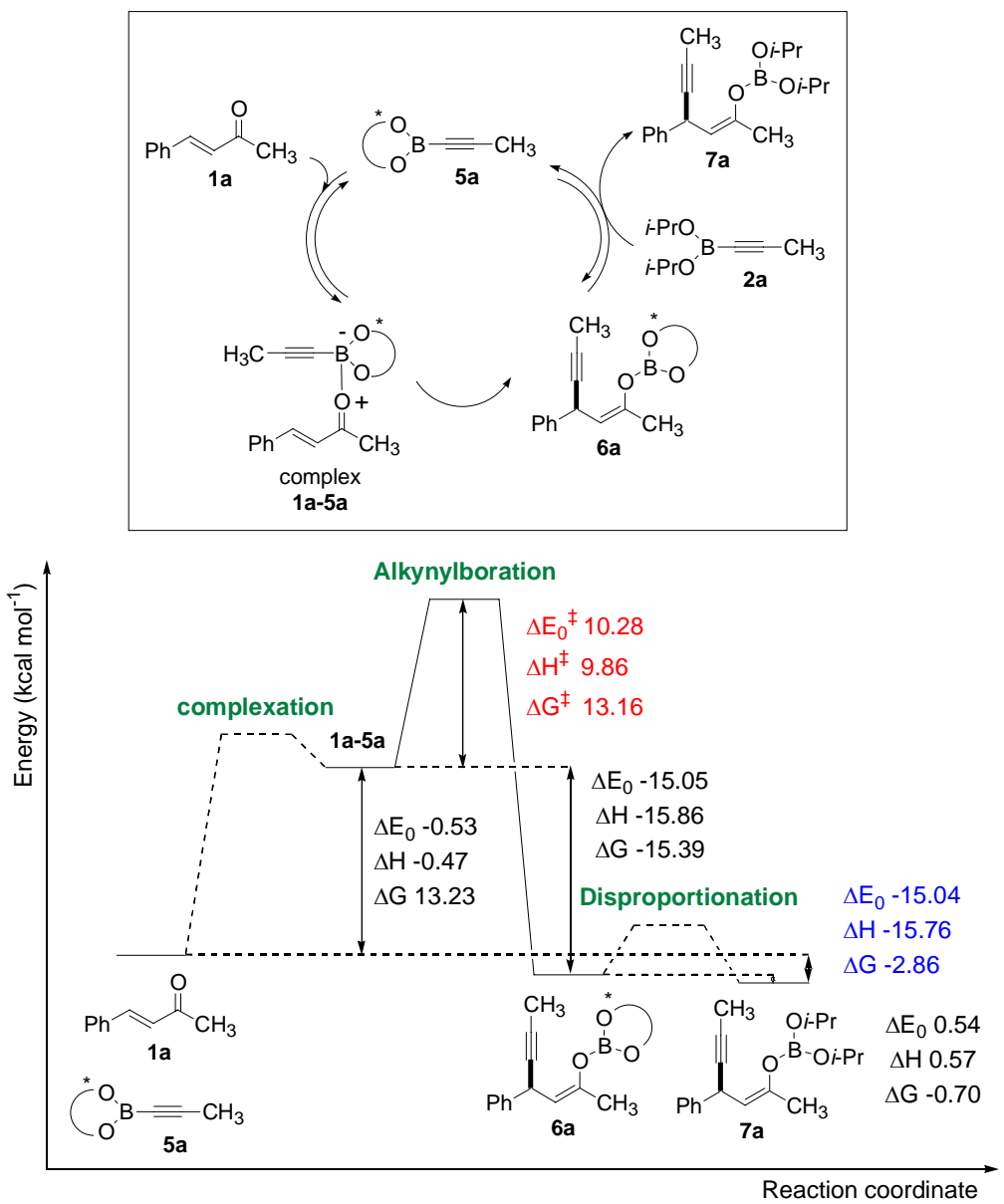

Scheme S2. B3LYP/lacvp* reaction coordinate of the conjugate addition of alkynylboronate 5a to enone 1a. All values in kcal $\mathrm{mol}^{-1}$. 
The reaction coordinate for the reaction of alkynylboronate 2a suggests that the C-C bond-forming reaction is slightly favored (Scheme S1). Although the enthalpic contribution of this reaction is very favorable $\left(-15.76 \mathrm{kcal} \mathrm{mol}^{-1}\right)$, the combination of two separate entities produces an loss of entropy, reducing the change in free energy of the system to $-2.86 \mathrm{kcal} \mathrm{mol}^{-1}$. In addition, the activation energy of this process is considerably high $\left(\Delta \mathrm{E}_{0}^{\ddagger} 26.06 \mathrm{kcal} \mathrm{mol}^{-1}, \Delta \mathrm{G}^{\ddagger} 41.35 \mathrm{kcal} \mathrm{mol}^{-1}\right)$, making it almost impossible for the reactants to surpass this barrier.

Due to the complexity of the coordination and the disproportionation in the reaction coordinate for the conjugate addition of alkynylboronate $\mathbf{5 a}$ to enone 1a, no attempts were made to calculate the activation energies of these steps (Scheme S2). However, the importance of the initial complexation step in the catalytic process is still quite evident. The formation of complex 1a-5a can be speculated to involve E/Z and s-trans/s-cis isomerism. However, these interconversion steps should involve small energy barriers, taking place rapidly and, possibly, in a reversible fashion. Once the complex is formed, the molecular orbitals of the reagents are affected in such a way that the activation energy of the conjugate addition is significantly reduced $\left(\Delta \mathrm{E}_{0}^{\ddagger} 10.28 \mathrm{kcal} \mathrm{mol}^{-1}, \Delta \mathrm{G}^{\ddagger} 13.16 \mathrm{kcal} \mathrm{mol}^{-1}\right)$. At the same time, since most of the entropy is lost with the initial formation of the complex, the free energy of the alkynylboration product (6a) is much lower than the complex $\left(\Delta \mathrm{G}=-15.39 \mathrm{kcal} \mathrm{mol}^{-1}\right)$. As a consequence the alkynylboration step should be irreversible since once $\mathbf{6 a}$ is formed, the energy barrier for going back to the complex is quite high (28.55 kcal mol${ }^{-1}$ ) so most molecules are likely to be trapped in the product.
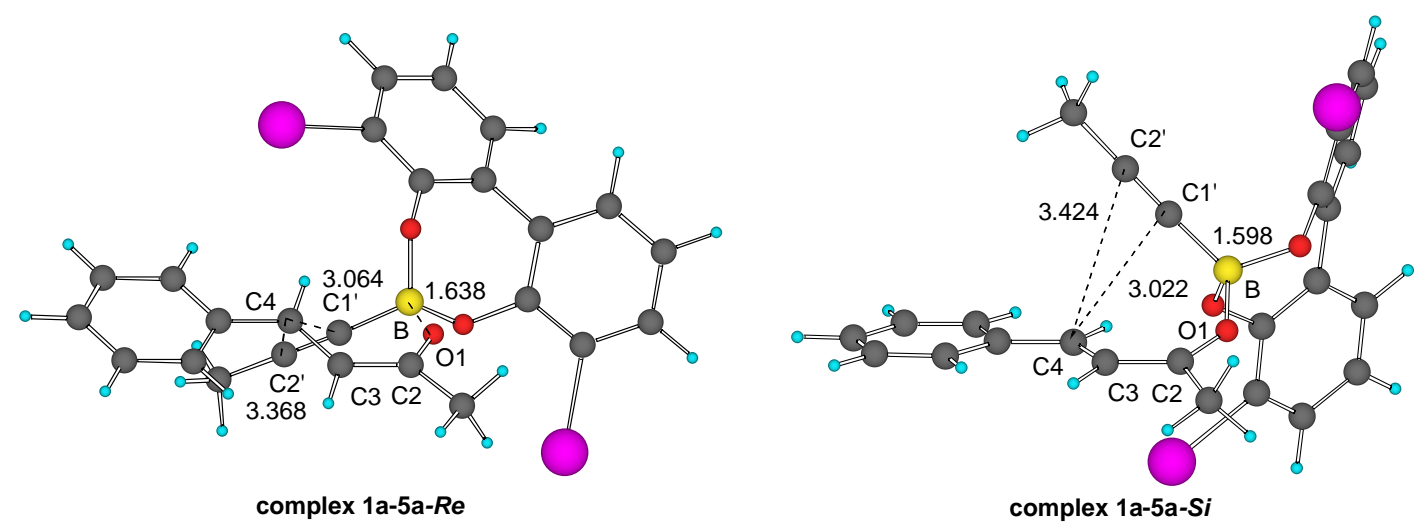

Figure S3. B3LYP/lacvp* optimized geometries of the complex between enone 1a and alkynylboronate 5a. Selected distances in $\AA$. 
Enone 1a

B3LYP/lacvp* Geometry

$\begin{array}{llll}\text { O1 } & 2.45581694 & -2.61149439 & 0.24368781 \\ \text { C2 } & 2.526537 & -1.40740683 & 0.03607523 \\ \text { C3 } & 3.86769831 & -0.71327863 & -0.13706258 \\ \text { C4 } & 1.31815562 & -0.54780097 & -0.05988475 \\ \text { C5 } & 0.08739598 & -1.07915165 & 0.0850188 \\ \text { C6 } & -1.20526433 & -0.39735485 & 0.02653597 \\ \text { C7 } & -2.37612495 & -1.15460655 & 0.21992197 \\ \text { C8 } & -1.33742333 & 0.9851596 & -0.21325361 \\ \text { C9 } & -3.63491632 & -0.55683293 & 0.17709229 \\ \text { C10 } & -2.593183 & 1.58235975 & -0.25673044 \\ \text { C11 } & -3.74773004 & 0.8145953 & -0.06142844 \\ \text { H12 } & 3.99416956 & 0.07308647 & 0.61840153 \\ \text { H13 } & 4.67483947 & -1.44247597 & -0.04439604 \\ \text { H14 } & 3.92406243 & -0.2256487 & -1.11899454 \\ \text { H15 } & 1.47036794 & 0.51293801 & -0.24618826 \\ \text { H16 } & 0.05410273 & -2.1525679 & 0.27103187 \\ \text { H17 } & -2.28871073 & -2.22256009 & 0.40580368 \\ \text { H18 } & -0.45119618 & 1.59407065 & -0.36755435 \\ \text { H19 } & -4.52576654 & -1.16020066 & 0.32923678 \\ \text { H20 } & -2.67575944 & 2.6498349 & -0.44325744 \\ \text { H21 } & -4.72685294 & 1.28476424 & -0.09609197\end{array}$

B3LYP/lacvp* Energy = -462.1178635

Number of Imaginary Frequencies $=0$

B-1-propynyldiisopropylboronate 2a B3LYP/lacvp* geometry

$\begin{array}{llll}\text { C1 } & 0.45218072 & 1.09662773 & -0.06533005 \\ \text { C2 } & 0.46664294 & 2.31140419 & -0.10344461 \\ \text { B3 } & 0.41018256 & -0.43808767 & -0.01108691 \\ \text { O4 } & 1.5313941 & -1.08856059 & 0.40596355 \\ \text { O5 } & -0.69737166 & -1.15924366 & -0.3645708 \\ \text { C6 } & 0.50500928 & 3.76998531 & -0.14286634 \\ \text { C7 } & 1.59438712 & -2.52275546 & 0.50493497 \\ \text { C8 } & -1.92526418 & -0.55823143 & -0.80835254 \\ \text { C9 } & 1.07941148 & -2.95716251 & 1.87663467 \\ \text { C10 } & 3.04729253 & -2.92044688 & 0.26455086 \\ \text { C11 } & -2.52318651 & -1.47474234 & -1.8711032 \\ \text { C12 } & -2.8477889 & -0.35982585 & 0.39294517 \\ \text { H13 } & 1.00886566 & 4.12306928 & -1.05122702 \\ \text { H14 } & -0.50446075 & 4.19901064 & -0.13253774 \\ \text { H15 } & 1.0520106 & 4.17369269 & 0.71790145 \\ \text { H16 } & 0.95607294 & -2.95764883 & -0.27335663 \\ \text { H17 } & -1.69667053 & 0.41936852 & -1.25030693 \\ \text { H18 } & 1.14599988 & -4.04574405 & 1.98785417 \\ \text { H19 } & 1.67303767 & -2.4897602 & 2.67068385 \\ \text { H20 } & 0.03304736 & -2.66194132 & 2.00331316 \\ \text { H21 } & 3.16298669 & -4.0090905 & 0.3134226 \\ \text { H22 } & 3.38145121 & -2.57860251 & -0.72051147 \\ \text { H23 } & 3.69623468 & -2.46635831 & 1.0219932 \\ \text { H24 } & -3.45569154 & -1.0528179 & -2.26252391 \\ \text { H25 } & -1.82446573 & -1.60480976 & -2.70403721 \\ \text { H26 } & -2.7398827 & -2.46195755 & -1.44715673 \\ \text { H27 } & -3.80393966 & 0.07499086 & 0.07921909\end{array}$

$\begin{array}{llll}\text { H28 } & -3.04719633 & -1.3196581 & 0.88337184\end{array}$

H29 $-2.38624332 \quad 0.31436582 \quad 1.12201134$

B3LYP/lacvp* Energy = -528.3066337

Number of Imaginary Frequencies $=0$

B-1-propynylboronate 5 a

B3LYP/lacvp* geometry

$\begin{array}{lrrr}\text { C1 } & 0.27620007 & 1.7828771 & -0.07262753 \\ \text { C2 } & 0.44464171 & 2.98574753 & -0.110635 \\ \text { B3 } & 0.06042029 & 0.28388242 & -0.02577547 \\ \text { O4 } & 0.75472668 & -0.45498337 & 0.90527864 \\ \text { O5 } & -0.82360902 & -0.2834188 & -0.91601373 \\ \text { C6 } & 0.64504104 & 4.42904714 & -0.15904032 \\ \text { C7 } & -1.60806406 & -1.373998 & -0.65216396 \\ \text { C8 } & 1.18105125 & -1.74347815 & 0.72435656 \\ \text { C9 } & -1.10068672 & -2.55504556 & -0.07261316 \\ \text { C10 } & -2.96408642 & -1.28944935 & -1.0016621 \\ \text { C11 } & 0.34313988 & -2.76093565 & 0.22324376 \\ \text { C12 } & 2.5059916 & -2.03676733 & 1.08031311 \\ \text { C13 } & -2.00463544 & -3.5992348 & 0.19221354 \\ \text { C14 } & -3.83555266 & -2.34897807 & -0.75598718 \\ \text { C15 } & 0.89809783 & -4.04013404 & 0.04046664 \\ \text { C16 } & 3.02551894 & -3.31971426 & 0.91730982 \\ \text { I17 } & -3.72451431 & 0.47527081 & -1.91185749 \\ \text { I18 } & 3.76113283 & -0.51442887 & 1.8730656 \\ \text { C19 } & -3.35130641 & -3.50469385 & -0.14333731 \\ \text { C20 } & 2.21684041 & -4.32204782 & 0.38178958 \\ \text { H21 } & -0.31462163 & 4.95992911 & -0.15394423 \\ \text { H22 } & 1.23457676 & 4.7787781 & 0.69673712 \\ \text { H23 } & 1.17813072 & 4.71348927 & -1.07464699 \\ \text { H24 } & -1.63696906 & -4.49338156 & 0.68494513 \\ \text { H25 } & -4.88201507 & -2.26480438 & -1.02826522 \\ \text { H26 } & 0.27880921 & -4.81879368 & -0.39268775 \\ \text { H27 } & 4.05357881 & -3.52765265 & 1.19325756 \\ \text { H28 } & -4.02762253 & -4.32583739 & 0.07502423 \\ \text { H29 } & 2.61998816 & -5.3185762 & 0.22759303\end{array}$

B3LYP/lacvp* Energy = -774.9100362

Number of Imaginary Frequencies $=0$

B-1-propynylboronate 9

B3LYP/lacvp* geometry

$\begin{array}{lrrr}\text { C1 } & -1.119873 & -0.009048 & 0.001022 \\ \text { C2 } & -2.333862 & -0.018311 & 0.000992 \\ \text { B3 } & 0.398071 & 0.0018 & 0.001038 \\ \text { O4 } & 1.159546 & -1.142349 & 0.001038 \\ \text { O5 } & 1.141541 & 1.157669 & -0.000916 \\ \text { C6 } & -3.792847 & -0.028427 & 0.000031 \\ \text { C7 } & 2.52597 & 0.789673 & -0.075821 \\ \text { C8 } & 2.538086 & -0.753876 & 0.07402 \\ \text { H9 } & -4.190216 & 0.604385 & -0.80336 \\ \text { H10 } & -4.1839 & -1.042633 & -0.141541 \\ \text { H11 } & -4.18544 & 0.355072 & 0.950058 \\ \text { H12 } & 3.07695 & 1.298889 & 0.720413 \\ \text { H13 } & 2.926132 & 1.120514 & -1.041428\end{array}$




\begin{tabular}{|c|c|c|c|}
\hline H14 & 3.095047 & -1.25502 & -0.724274 \\
\hline H15 & 2.944855 & -1.078232 & 1.038666 \\
\hline \multicolumn{4}{|c|}{ B3LYP/lacvp* Energy = -369.9628173 } \\
\hline \multicolumn{4}{|c|}{ B-1-propynylboronate 10} \\
\hline C1 & -2.587189 & -0.006409 & 0.00024 \\
\hline C2 & -3.801208 & -0.008347 & 0.000122 \\
\hline B3 & -1.068222 & -0.00354 & 0.000244 \\
\hline $\mathrm{O} 4$ & -0.312057 & -1.149872 & 0.000244 \\
\hline $\mathrm{O} 5$ & -0.316406 & 1.146622 & -0.000107 \\
\hline C6 & -5.260239 & -0.009598 & 0.000656 \\
\hline C7 & 1.066711 & 0.757523 & -0.227097 \\
\hline C8 & 1.069839 & -0.753983 & 0.226715 \\
\hline C9 & 1.96817 & 1.684006 & 0.586533 \\
\hline C10 & 1.332306 & 0.944031 & -1.726913 \\
\hline C11 & 1.337265 & -0.939926 & 1.726669 \\
\hline C12 & 1.974442 & -1.677032 & -0.588013 \\
\hline H13 & -5.65419 & 0.58078 & -0.836868 \\
\hline H14 & -5.658997 & -1.027328 & -0.085495 \\
\hline H15 & -5.651596 & 0.427856 & 0.92755 \\
\hline H16 & 3.019836 & 1.392395 & 0.486465 \\
\hline H17 & 1.864212 & 2.710968 & 0.219849 \\
\hline H18 & 1.701813 & 1.676331 & 1.646393 \\
\hline H19 & 2.37822 & 0.740143 & -1.98080 \\
\hline $\mathrm{H} 20$ & 0.693527 & 0.288086 & -2.327759 \\
\hline H21 & 1.105423 & 1.978958 & -2.000183 \\
\hline $\mathrm{H} 22$ & 2.381363 & -0.730576 & 1.980042 \\
\hline $\mathrm{H} 23$ & 0.695084 & -0.288223 & 2.3281 \\
\hline $\mathrm{H} 24$ & 1.115158 & -1.977051 & 1.99934 \\
\hline $\mathrm{H} 25$ & 3.02475 & -1.380173 & -0.489471 \\
\hline H26 & 1.875381 & -2.703995 & -0.22038 \\
\hline $\mathrm{H} 27$ & 1.706802 & -1.671371 & -1.6464 \\
\hline
\end{tabular}

B3LYP/lacvp* Energy $=-527.1193852$

Number of Imaginary Frequencies $=0$

\section{TSs of Conjugate Additions to Enone 1a}

\section{Conjugate Addition of 2a to 1a \\ TS 1a+2a \\ B3LYP/lacvp* Geometry}

$\begin{array}{lrrr}\text { O1 } & 0.01709116 & -2.34344325 & 0.45068515 \\ \text { B2 } & 0.15199614 & -0.93762095 & 0.48389951 \\ \text { C3 } & -1.09400393 & -2.97295244 & -0.16988924 \\ \text { C4 } & 0.18203374 & -0.23410052 & -1.05260583 \\ \text { O5 } & 1.34222691 & -0.47938186 & 1.12394138 \\ \text { C6 } & -2.04206153 & -3.52162042 & 0.89966017 \\ \text { C7 } & -0.56302868 & -4.07414113 & -1.09048312 \\ \text { C8 } & 0.28488691 & -0.37770396 & -2.27702327 \\ \text { C9 } & 1.9471687 & -1.22872403 & 2.1742892 \\ \text { C10 } & 0.42334318 & -0.41673213 & -3.72076434 \\ \text { C11 } & 3.42341655 & -0.8369146 & 2.21564887 \\ \text { C12 } & 1.24017272 & -0.95990774 & 3.50674798 \\ \text { O13 } & -1.11204637 & -0.33609107 & 1.14558085\end{array}$

$\begin{array}{lrrr}\text { C14 } & -1.60004191 & 0.85791117 & 0.94478038 \\ \text { C15 } & 0.24110967 & 1.6106468 & -0.52638838 \\ \text { C16 } & -2.92655119 & 1.09687224 & 1.6157786 \\ \text { C17 } & -1.02670374 & 1.80607304 & 0.12123503 \\ \text { C18 } & 0.63661082 & 2.53080187 & -1.62873682 \\ \text { C19 } & 1.98582186 & 2.89634911 & -1.76292139 \\ \text { C20 } & -0.29892593 & 3.05944258 & -2.53490519 \\ \text { C21 } & 2.38858193 & 3.78573767 & -2.75995097 \\ \text { C22 } & 0.1029529 & 3.9456165 & -3.53504 \\ \text { C23 } & 1.44763023 & 4.31464221 & -3.64772212 \\ \text { H24 } & -1.64247843 & -2.23967263 & -0.78152521 \\ \text { H25 } & -2.40226721 & -2.70749557 & 1.53532457 \\ \text { H26 } & -1.51553257 & -4.24565624 & 1.5328497 \\ \text { H27 } & -2.90524797 & -4.02293391 & 0.4436659 \\ \text { H28 } & 0.01220421 & -4.80426937 & -0.50915739 \\ \text { H29 } & 0.10211174 & -3.64833052 & -1.84972107 \\ \text { H30 } & -1.38189629 & -4.59962811 & -1.59707087 \\ \text { H31 } & 1.86330156 & -2.2970129 & 1.93849702 \\ \text { H32 } & 0.49730491 & 0.61561263 & -4.09687507 \\ \text { H33 } & 1.33117386 & -0.94868373 & -4.03055912 \\ \text { H34 } & -0.44248131 & -0.88445647 & -4.20502857 \\ \text { H35 } & 3.52734207 & 0.23396872 & 2.43088501 \\ \text { H36 } & 3.89703893 & -1.03835126 & 1.24876433 \\ \text { H37 } & 3.95901186 & -1.39781921 & 2.99066647 \\ \text { H38 } & 0.18726971 & -1.25280004 & 3.44761662 \\ \text { H39 } & 1.28645051 & 0.10840719 & 3.75220905 \\ \text { H40 } & 1.71145578 & -1.52399691 & 4.32147223 \\ \text { H41 } & 1.075113 & 1.33952417 & 0.12027099 \\ \text { H42 } & -3.66091 & 0.3604529 & 1.26756638 \\ \text { H43 } & -2.82217131 & 0.95907645 & 2.6982284 \\ \text { H44 } & -3.30579879 & 2.10355537 & 1.42174474 \\ \text { H45 } & -1.62177975 & 2.67362938 & -0.14266306 \\ \text { H46 } & 2.71783977 & 2.48189801 & -1.07390179 \\ \text { H47 } & -1.34267783 & 2.7666304 & -2.45680885 \\ \text { H48 } & 3.4354128 & 4.06549188 & -2.84348732 \\ \text { H49 } & -0.63209678 & 4.34838121 & -4.22718416 \\ \text { H50 } & 1.7596048 & 5.00677185 & -4.42554833 \\ & & & \\ \end{array}$

B3LYP/6-31G* Energy $=-990.3829662$

Number of Imaginary Frequencies $=1(-350.00)$

\section{Complex 1a-5a-Re}

B3LYP/lacvP* Geometry

$\begin{array}{lrrr}\text { O1 } & -0.47108664 & -2.83147592 & -0.7920322 \\ \text { B2 } & -0.03821049 & -1.47519645 & -0.64154023 \\ \text { C3 } & -1.76993884 & -3.19289655 & -0.74685621 \\ \text { C4 } & 1.52265513 & -1.42856215 & -0.5207412 \\ \text { O5 } & -0.77741012 & -0.6709507 & 0.31804397 \\ \text { C6 } & -2.55734169 & -2.96396251 & 0.40668401 \\ \text { C7 } & -2.33424746 & -3.89989027 & -1.82127386 \\ \text { C8 } & 2.72678479 & -1.41566186 & -0.35527743 \\ \text { C9 } & -1.13912915 & -1.17941879 & 1.51573281 \\ \text { C10 } & -1.99062652 & -2.3095322 & 1.61376531 \\ \text { C11 } & -3.88720178 & -3.41381477 & 0.41810149 \\ \text { C12 } & -3.65011538 & -4.35662775 & -1.78974314 \\ \text { I13 } & -1.14114441 & -4.31733167 & -3.54055472 \\ \text { C14 } & 4.17312718 & -1.397523 & -0.14863613\end{array}$




$\begin{array}{lrrr}\text { C15 } & -0.74925883 & -0.53654931 & 2.70258739 \\ \text { C16 } & -2.34917184 & -2.78217961 & 2.88605721 \\ \text { C17 } & -4.43331916 & -4.10215028 & -0.66246591 \\ \text { C18 } & -1.12934068 & -1.00322308 & 3.95932873 \\ \text { C19 } & -1.9247049 & -2.14590293 & 4.04956462 \\ \text { I20 } & 0.42230811 & 1.24390984 & 2.60120772 \\ \text { H21 } & -4.50011033 & -3.20618387 & 1.29025977 \\ \text { H22 } & -4.05936918 & -4.89869979 & -2.63569847 \\ \text { H23 } & 4.60334522 & -0.42668467 & -0.42755003 \\ \text { H24 } & 4.42131436 & -1.57856997 & 0.90484124 \\ \text { H25 } & 4.67353817 & -2.16960868 & -0.74622196 \\ \text { H26 } & -2.9652214 & -3.67387032 & 2.9541182 \\ \text { H27 } & -5.4653332 & -4.43994248 & -0.63298012 \\ \text { H28 } & -0.80242651 & -0.48543322 & 4.85481777 \\ \text { H29 } & -2.20970211 & -2.53272934 & 5.02379111 \\ \text { O30 } & -0.53541533 & -0.76869266 & -2.03379075 \\ \text { C31 } & -0.13334509 & 0.00947905 & -2.93842043 \\ \text { C32 } & 1.45099718 & 1.419557 & -1.64697598 \\ \text { C33 } & -0.83853853 & -0.14102852 & -4.2624308 \\ \text { C34 } & 0.90745424 & 0.99943748 & -2.82290335 \\ \text { C35 } & 2.52686607 & 2.39279246 & -1.50210745 \\ \text { C36 } & 3.0303001 & 3.16046687 & -2.5744666 \\ \text { C37 } & 3.10292819 & 2.55892229 & -0.22545805 \\ \text { C38 } & 4.07119805 & 4.06017392 & -2.37183766 \\ \text { C39 } & 4.15223132 & 3.45431748 & -0.0270588 \\ \text { C40 } & 4.6368096 & 4.20905746 & -1.09856274 \\ \text { H41 } & 1.05640279 & 1.01557872 & -0.72066356 \\ \text { H42 } & -1.91028475 & 0.03485422 & -4.11548738 \\ \text { H43 } & -0.45982998 & 0.54397474 & -5.02382007 \\ \text { H44 } & -0.73500269 & -1.17909464 & -4.6020573 \\ \text { H45 } & 1.24289673 & 1.42278468 & -3.76463594 \\ \text { H46 } & 2.59663722 & 3.06475878 & -3.56533958 \\ \text { H47 } & 2.72347257 & 1.96493085 & 0.60143329 \\ \text { H48 } & 4.44404271 & 4.65068734 & -3.20418202 \\ \text { H49 } & 4.58839721 & 3.56578455 & 0.96168418 \\ \text { H50 } & 5.45008054 & 4.91352138 & -0.94542859\end{array}$

B3LYP/lacvp* Energy = -1237.028366

Number of Imaginary Frequencies $=0$

Complex 1a-5a-Si

B3LYP/lacvp* Geometry

$\begin{array}{lrrr}\text { O1 } & -1.99588464 & -0.30247748 & -0.99219047 \\ \text { B2 } & -2.17355205 & 1.11496696 & -0.69601928 \\ \text { C3 } & -2.96635422 & -1.23729337 & -0.91796601 \\ \text { C4 } & -0.89754467 & 1.69885031 & 0.01111271 \\ \text { O5 } & -3.43089394 & 1.44310799 & -0.06094274 \\ \text { C6 } & -3.82342067 & -1.36432608 & 0.20754536 \\ \text { C7 } & -3.07624328 & -2.19433038 & -1.94316588 \\ \text { O8 } & -2.38857728 & 1.85382995 & -2.09563914 \\ \text { C9 } & 0.0713677 & 2.13620573 & 0.60053129 \\ \text { C10 } & -3.62375343 & 0.95020192 & 1.18297554 \\ \text { C11 } & -3.76485894 & -0.4441061 & 1.37301821 \\ \text { C12 } & -4.00100425 & -3.23466804 & -1.89977119 \\ \text { C13 } & -4.76118776 & -2.40960551 & 0.2288824 \\ \text { I14 } & -1.77594059 & -2.08341284 & -3.63513288 \\ \text { C15 } & -1.64369041 & 2.31385305 & -3.00841773\end{array}$

\begin{tabular}{lrrr} 
C16 & 0.43398879 & 1.01731698 & -2.61491633 \\
C17 & 1.22733395 & 2.66939453 & 1.31767492 \\
C18 & -4.85643533 & -3.33832077 & -0.8031792 \\
C19 & -3.7466866 & 1.80327044 & 2.29073836 \\
C20 & -3.94113818 & -0.93847895 & 2.67494278 \\
C21 & -2.33643757 & 3.26503694 & -3.95098955 \\
C22 & -0.25087517 & 2.03467021 & -3.21586088 \\
C23 & 1.84496772 & 0.70692555 & -2.78485785 \\
C24 & -3.9442283 & 1.30058651 & 3.57609626 \\
C25 & -4.02313809 & -0.08018803 & 3.76877456 \\
I26 & -3.66537546 & 3.91469981 & 2.01251514 \\
C27 & 2.33732096 & -0.46723281 & -2.17719989 \\
C28 & 2.74170009 & 1.52088837 & -3.51063588 \\
C29 & 3.67860595 & -0.82347829 & -2.29791568 \\
C30 & 4.08028038 & 1.16432676 & -3.62816957 \\
C31 & 4.55201086 & -0.00926065 & -3.02436803 \\
H32 & -4.0572431 & -3.94785483 & -2.71517792 \\
H33 & -5.43923602 & -2.47501766 & 1.07441374 \\
H34 & -0.11657039 & 0.32917746 & -1.98453079 \\
H35 & 2.07016751 & 2.85044723 & 0.63799226 \\
H36 & 1.57070146 & 1.9708365 & 2.09114525 \\
H37 & 0.98457631 & 3.61903858 & 1.81062137 \\
H38 & -5.59531641 & -4.13342406 & -0.76248723 \\
H39 & -4.00573916 & -2.0123773 & 2.82445306 \\
H40 & -2.66667478 & 4.14683261 & -3.38860648 \\
H41 & -3.23687094 & 2.78254811 & -4.34572096 \\
H42 & -1.69663097 & 3.57865467 & -4.77837836 \\
H43 & 0.24198435 & 2.67606558 & -3.93989252 \\
H44 & -4.02580344 & 1.97932769 & 4.41861888 \\
H45 & -4.15187973 & -0.47958374 & 4.77095477 \\
H46 & 1.65168568 & -1.09418581 & -1.61370076 \\
H47 & 2.39180107 & 2.43836477 & -3.97440245 \\
& 4.04263019 & -1.73169122 & -1.82621237 \\
H49 & 4.76212607 & 1.79928182 & -4.18709232 \\
\hline & & -0.28336054 & -3.11878809
\end{tabular}

B3LYP/lacvp* Energy = -1237.02875 Number of Imaginary Frequencies $=0$

\section{Conjugate Addition of 5a to 1a TS 1a+5a-Re \\ B3LYP/lacvp* Geometry}

$\begin{array}{lrrr}\text { O1 } & -0.72760026 & -2.06652504 & -0.8470748 \\ \text { B2 } & -0.70917598 & -0.62429214 & -0.82219038 \\ \text { C3 } & -1.92119839 & -2.69991536 & -0.79427982 \\ \text { C4 } & 0.87576148 & -0.16187113 & -0.83850829 \\ \text { O5 } & -1.38239237 & -0.01602956 & 0.32418063 \\ \text { O6 } & -1.39058293 & -0.09636185 & -2.03944484 \\ \text { C7 } & -2.70706823 & -2.63898464 & 0.37848948 \\ \text { C8 } & -2.36261852 & -3.4862084 & -1.86913319 \\ \text { C9 } & 2.03197082 & -0.49172749 & -0.53918495 \\ \text { C10 } & -0.94796498 & 0.80471604 & -2.88201513 \\ \text { C11 } & 0.78827774 & 1.65782095 & -1.34257635 \\ \text { C12 } & -1.58841257 & -0.64458292 & 1.49774484 \\ \text { C13 } & -2.22304476 & -1.91373087 & 1.58114304 \\ \text { C14 } & -3.92912303 & -3.32840415 & 0.41312638\end{array}$




$\begin{array}{lrrr}\text { C15 } & -3.56830306 & -4.18325224 & -1.81555039 \\ \text { I16 } & -1.15532216 & -3.64004257 & -3.62062284 \\ \text { C17 } & 3.42216052 & -0.73700331 & -0.22151412 \\ \text { C18 } & -1.6718119 & 0.81136441 & -4.19721311 \\ \text { C19 } & 0.12433595 & 1.6251123 & -2.6227422 \\ \text { C20 } & 2.08697377 & 2.39217772 & -1.26516471 \\ \text { C21 } & -1.26341477 & 0.00612475 & 2.70260806 \\ \text { C22 } & -2.44073338 & -2.48404423 & 2.84546688 \\ \text { C23 } & -4.35981495 & -4.09261907 & -0.66879362 \\ \text { C24 } & 3.03390204 & 2.32415376 & -2.30225142 \\ \text { C25 } & 2.36146479 & 3.20444223 & -0.15423024 \\ \text { C26 } & -1.5026816 & -0.56388732 & 3.95105599 \\ \text { C27 } & -2.08641077 & -1.82841924 & 4.02108071 \\ \text { I28 } & -0.4154712 & 1.96460515 & 2.65524882 \\ \text { C29 } & 4.21425313 & 3.06503743 & -2.23565792 \\ \text { C30 } & 3.53878606 & 3.95175271 & -0.08991793 \\ \text { C31 } & 4.46741178 & 3.88625969 & -1.1314585 \\ \text { H32 } & 0.15292054 & 1.84133349 & -0.47540244 \\ \text { H33 } & -4.55022241 & -3.25004698 & 1.30062099 \\ \text { H34 } & -3.88973828 & -4.78171336 & -2.66138223 \\ \text { H35 } & 4.00155896 & 0.15968463 & -0.49271886 \\ \text { H36 } & 3.57026286 & -0.9225585 & 0.84875465 \\ \text { H37 } & 3.82655763 & -1.57981447 & -0.79447251 \\ \text { H38 } & -2.73954957 & 0.99299937 & -4.0285361 \\ \text { H39 } & -1.28137245 & 1.5787739 & -4.87018972 \\ \text { H40 } & -1.58440448 & -0.17438749 & -4.66997673 \\ \text { H41 } & 0.54615754 & 2.19298046 & -3.44399315 \\ \text { H42 } & -2.88947095 & -3.47159006 & 2.89540347 \\ \text { H43 } & -5.31038306 & -4.61669273 & -0.6257748 \\ \text { H44 } & 2.84287899 & 1.68416442 & -3.15970964 \\ \text { H45 } & 1.64218169 & 3.24942147 & 0.65829744 \\ \text { H46 } & -1.23157689 & -0.02903272 & 4.85505794 \\ \text { H47 } & -2.26191237 & -2.29363896 & 4.98679519 \\ \text { H48 } & 4.936083 & 3.00434462 & -3.04589003 \\ \text { H49 } & 3.72871514 & 4.58487706 & 0.77272443 \\ \text { H50 } & 5.38425822 & 4.46795405 & -1.08318499\end{array}$

B3LYP/6-31G* Energy = -1237.010503

Number of Imaginary Frequencies $=1(-289.47)$

TS 1a+5a-Si

B3LYP/lacvp* Geometry

$\begin{array}{lrrr}\text { O1 } & -1.58713423 & -0.62271146 & -0.80980846 \\ \text { B2 } & -1.63262448 & 0.8337727 & -0.69767355 \\ \text { C3 } & -2.67854412 & -1.40067997 & -0.64516491 \\ \text { C4 } & -0.06785729 & 1.25195111 & -0.35499126 \\ \text { O5 } & -2.57233796 & 1.34210721 & 0.27253049 \\ \text { C6 } & -3.40142739 & -1.40476725 & 0.57288789 \\ \text { C7 } & -3.05732824 & -2.30148743 & -1.65442543 \\ \text { O8 } & -2.02211297 & 1.47357854 & -1.9781461 \\ \text { C9 } & 0.7505101 & 1.64629421 & 0.48566477 \\ \text { C10 } & -2.63515486 & 0.81419218 & 1.51122848 \\ \text { C11 } & -3.0099226 & -0.53783182 & 1.71337858 \\ \text { C12 } & -4.13174736 & -3.17461177 & -1.49703429 \\ \text { C13 } & -4.49175182 & -2.27785763 & 0.71168048 \\ \text { I14 } & -1.95183839 & -2.35841645 & -3.47847611 \\ \text { C15 } & -1.20672401 & 1.82031997 & -2.94821679\end{array}$

$\begin{array}{lrrr}\text { C16 } & 0.78601102 & 0.71995026 & -1.97136456 \\ \text { C17 } & 1.80312656 & 2.10431445 & 1.36814081 \\ \text { C18 } & -4.85823434 & -3.15527839 & -0.30563216 \\ \text { C19 } & -2.4067331 & 1.62285815 & 2.63796718 \\ \text { C20 } & -3.07321379 & -1.03722895 & 3.0233152 \\ \text { C21 } & -1.87072644 & 2.62830301 & -4.02574713 \\ \text { C22 } & 0.14130584 & 1.54740364 & -2.96074124 \\ \text { C23 } & 2.27461814 & 0.70953262 & -1.90608705 \\ \text { C24 } & -2.49161807 & 1.11835677 & 3.93489661 \\ \text { C25 } & -2.81237263 & -0.22654687 & 4.1256311 \\ \text { I26 } & -1.97150117 & 3.69761452 & 2.37592206 \\ \text { C27 } & 2.93685849 & -0.49108114 & -1.60202829 \\ \text { C28 } & 3.04152599 & 1.86039391 & -2.15775428 \\ \text { C29 } & 4.3307127 & -0.54799118 & -1.5712451 \\ \text { C30 } & 4.43559994 & 1.80525787 & -2.12292036 \\ \text { C31 } & 5.08381837 & 0.599965 & -1.83308885 \\ \text { H32 } & -4.40214299 & -3.85500374 & -2.29753464 \\ \text { H33 } & -5.06555669 & -2.25078865 & 1.63333824 \\ \text { H34 } & 0.35994954 & -0.27264562 & -1.83263521 \\ \text { H35 } & 2.72924079 & 2.21704145 & 0.78349319 \\ \text { H36 } & 1.99650004 & 1.39388204 & 2.1799984 \\ \text { H37 } & 1.55423839 & 3.08156302 & 1.79952856 \\ \text { H38 } & -5.70856734 & -3.81916912 & -0.17869771 \\ \text { H39 } & -3.32329281 & -2.08388992 & 3.1704376 \\ \text { H40 } & -2.34027881 & 3.5183314 & -3.5908538 \\ \text { H41 } & -2.66854871 & 2.03468013 & -4.48703796 \\ \text { H42 } & -1.16121664 & 2.93066152 & -4.79993586 \\ \text { H43 } & 0.75538662 & 2.05458632 & -3.69634243 \\ \text { H44 } & -2.30512356 & 1.76654623 & 4.78472385 \\ \text { H45 } & -2.86193781 & -0.63422863 & 5.1314092 \\ \text { H46 } & 2.35075214 & -1.38416621 & -1.39878812 \\ \text { H47 } & 2.54124081 & 2.8005493 & -2.37480304 \\ \text { H48 } & 4.82793039 & -1.48721978 & -1.34428822 \\ \text { H49 } & 5.01670369 & 2.70186312 & -2.32219206 \\ \text { H50 } & 6.16959577 & 0.55736923 & -1.80894765\end{array}$

B3LYP/6-31G* Energy = -1237.012374

Number of Imaginary Frequencies $=1(-301.36)$

\section{Conjugate Addition of 9 to 1a} TS $1 \mathrm{a}+9$

B3LYP/lacvp* Geometry

$\begin{array}{lrrr}\text { O1 } & -1.95276808 & -1.33100461 & -0.35994442 \\ \text { B2 } & -1.98656386 & 0.12057397 & -0.32600929 \\ \text { C3 } & -3.2881364 & -1.78859102 & -0.20994057 \\ \text { C4 } & -0.86909681 & 0.60396754 & 0.82933506 \\ \text { O5 } & -3.32080856 & 0.53978482 & -0.04500404 \\ \text { O6 } & -1.53934267 & 0.75022929 & -1.61834843 \\ \text { C7 } & -0.60618443 & 1.07667297 & 1.94106248 \\ \text { C8 } & -4.0243083 & -0.59486266 & 0.42650865 \\ \text { C9 } & -0.30175418 & 0.96818493 & -1.97581348 \\ \text { C10 } & 0.69564339 & -0.16374914 & -0.01699015 \\ \text { C11 } & -0.17692927 & 1.59786582 & 3.2252232 \\ \text { C12 } & -0.169917 & 1.76112613 & -3.24692558 \\ \text { C13 } & 0.7990417 & 0.59732324 & -1.23080106 \\ \text { C14 } & 1.88360802 & -0.26217332 & 0.87400765 \\ \text { C15 } & 2.13263415 & -1.46752904 & 1.55061912\end{array}$




$\begin{array}{lrrr}\text { C16 } & 2.78389836 & 0.8042786 & 1.04220639 \\ \text { C17 } & 3.26465138 & -1.61487942 & 2.35290307 \\ \text { C18 } & 3.91327414 & 0.65987751 & 1.84852618 \\ \text { C19 } & 4.15971438 & -0.55206551 & 2.50205437 \\ \text { H20 } & -3.30972148 & -2.6910389 & 0.41474639 \\ \text { H21 } & -3.71751301 & -2.03853046 & -1.19296345 \\ \text { H22 } & -3.97762492 & -0.65041139 & 1.52759281 \\ \text { H23 } & -5.07907854 & -0.53029736 & 0.13226765 \\ \text { H24 } & 0.13421194 & -1.09591749 & -0.07035701 \\ \text { H25 } & 0.91520496 & 1.48348073 & 3.30745708 \\ \text { H26 } & -0.63187028 & 1.05527033 & 4.06277032 \\ \text { H27 } & -0.40639846 & 2.66521494 & 3.33037091 \\ \text { H28 } & -0.67664445 & 2.72730945 & -3.13720469 \\ \text { H29 } & -0.66751743 & 1.22936976 & -4.06602298 \\ \text { H30 } & 0.87624569 & 1.92848184 & -3.5151656 \\ \text { H31 } & 1.76230095 & 1.00914595 & -1.51108461 \\ \text { H32 } & 1.43595184 & -2.2943289 & 1.43456692 \\ \text { H33 } & 2.59138309 & 1.74994979 & 0.54248083 \\ \text { H34 } & 3.44872768 & -2.55855139 & 2.85953037 \\ \text { H35 } & 4.60254644 & 1.4919659 & 1.96658676 \\ \text { H36 } & 5.04231409 & -0.6651549 & 3.12620291\end{array}$

B3LYP/6-31G* Energy = -832.0435878

Number of Imaginary Frequencies $=1(-342.74)$

\section{Conjugate Addition of 10 to 1a TS $1 \mathrm{a}+10$ \\ B3LYP/lacvp* Geometry}

$\begin{array}{lrrr}\text { O1 } & -1.93926961 & -1.33799202 & -0.26480075 \\ \text { B2 } & -1.98227569 & 0.10889966 & -0.27489012 \\ \text { C3 } & -0.83949312 & 0.63891739 & 0.8336799 \\ \text { O4 } & -3.31456302 & 0.52294614 & 0.00750497 \\ \text { O5 } & -1.55470162 & 0.70816728 & -1.59834122 \\ \text { C6 } & -0.56470658 & 1.16815065 & 1.91651447 \\ \text { C7 } & -0.32364653 & 0.91761768 & -1.98241741 \\ \text { C8 } & 0.70009027 & -0.16409039 & -0.01330055 \\ \text { C9 } & -0.12954781 & 1.74723335 & 3.17346385 \\ \text { C10 } & -0.21302487 & 1.67973145 & -3.27449268 \\ \text { C11 } & 0.79056128 & 0.56533937 & -1.24714206 \\ \text { C12 } & 1.89132641 & -0.25499838 & 0.87371624 \\ \text { C13 } & 2.11357483 & -1.44136723 & 1.59234408 \\ \text { C14 } & 2.81270259 & 0.7978037 & 1.00742024 \\ \text { C15 } & 3.23881755 & -1.58393312 & 2.40463901 \\ \text { C16 } & 3.93623237 & 0.65809739 & 1.82304659 \\ \text { C17 } & 4.15481586 & -0.53469982 & 2.52052253 \\ \text { H18 } & 0.13177405 & -1.0928928 & -0.03973297 \\ \text { H19 } & 0.95516259 & 1.59071167 & 3.28054636 \\ \text { H20 } & -0.61930843 & 1.27357781 & 4.03313802 \\ \text { H21 } & -0.31487291 & 2.82743294 & 3.21333287 \\ \text { H22 } & -0.71823243 & 2.64834768 & -3.17998861 \\ \text { H23 } & -0.72354297 & 1.12836756 & -4.07251082 \\ \text { H24 } & 0.82857976 & 1.84062473 & -3.56383569 \\ \text { H25 } & 1.74939196 & 0.97085441 & -1.55109193 \\ \text { H26 } & 1.398611 & -2.25591209 & 1.50371324 \\ \text { H27 } & 2.64103482 & 1.72960649 & 0.47509012 \\ \text { H28 } & 3.40044443 & -2.51242772 & 2.94586005 \\ \text { H29 } & 4.64211214 & 1.47943142 & 1.91525252\end{array}$

$\begin{array}{lrrr}\text { H30 } & 5.03179051 & -0.64333756 & 3.15334204 \\ \text { C31 } & -3.29431934 & -1.81201352 & -0.2405913 \\ \text { C32 } & -4.05064783 & -0.61984265 & 0.46238864 \\ \text { C33 } & -3.33093578 & -3.14124496 & 0.51695964 \\ \text { C34 } & -3.74949016 & -2.02864811 & -1.69415591 \\ \text { C35 } & -3.95858656 & -0.68352013 & 1.99871195 \\ \text { C36 } & -5.51353444 & -0.44829016 & 0.04744272 \\ \text { H37 } & -4.35913864 & -3.50741094 & 0.62509687 \\ \text { H38 } & -2.76015411 & -3.89670406 & -0.03496539 \\ \text { H39 } & -2.88828863 & -3.04522402 & 1.51175351 \\ \text { H40 } & -4.75178486 & -2.46895558 & -1.75020326 \\ \text { H41 } & -3.74877434 & -1.08480407 & -2.24731485 \\ \text { H42 } & -3.04602773 & -2.71024377 & -2.18451051 \\ \text { H43 } & -4.58488623 & -1.48088908 & 2.41530242 \\ \text { H44 } & -2.92701441 & -0.84436905 & 2.32560805 \\ \text { H45 } & -4.29978635 & 0.27275948 & 2.4097751 \\ \text { H46 } & -6.10631716 & -1.33328577 & 0.30964565 \\ \text { H47 } & -5.94340985 & 0.41424268 & 0.56872483 \\ \text { H48 } & -5.60314767 & -0.26994929 & -1.02670151\end{array}$

B3LYP/6-31G* Energy $=-989.1990118$ Number of Imaginary Frequencies $=1(-345.91)$

\section{TSs of Hetero Diels-Alder Reactions}

\section{Hetero Diels-Alder Reaction between 1a and 2a DATS 1a+2a-A \\ B3LYP/lacvP* Geometry}

$\begin{array}{lrrr}\text { C1 } & 1.05229117 & -0.72626373 & -0.31500882 \\ \text { C2 } & -0.19626977 & -0.88391663 & -0.23135539 \\ \text { B3 } & 2.57099872 & -0.75416966 & -0.59914116 \\ \text { C4 } & -1.26138087 & -1.74702976 & -0.81822324 \\ \text { O5 } & 3.20398921 & 0.17456286 & -1.37264464 \\ \text { O6 } & 3.26933329 & -1.8211171 & -0.11361678 \\ \text { C7 } & 2.58257677 & 1.35879072 & -1.89406082 \\ \text { C8 } & 4.69274375 & -1.93055984 & -0.29532948 \\ \text { C9 } & 2.80884704 & 1.38135802 & -3.4037673 \\ \text { C10 } & 3.17813955 & 2.57152562 & -1.18274879 \\ \text { C11 } & 5.40489103 & -1.16695484 & 0.82073566 \\ \text { C12 } & 5.02741954 & -3.41852436 & -0.30411206 \\ \text { H13 } & -2.00769189 & -1.14577589 & -1.3495942 \\ \text { H14 } & -0.83411772 & -2.47310533 & -1.51669471 \\ \text { H15 } & -1.79717005 & -2.29244843 & -0.03318362 \\ \text { H16 } & 1.50650776 & 1.31416155 & -1.6871154 \\ \text { H17 } & 4.95851442 & -1.48481547 & -1.26110596 \\ \text { H18 } & 2.36571388 & 0.49727257 & -3.87511521 \\ \text { H19 } & 3.88168062 & 1.38848173 & -3.6284993 \\ \text { H20 } & 2.35563945 & 2.27495643 & -3.84834056 \\ \text { H21 } & 4.26095565 & 2.61761025 & -1.34915836 \\ \text { H22 } & 2.98585775 & 2.50061102 & -0.10921998 \\ \text { H23 } & 2.73034867 & 3.49774643 & -1.56259706 \\ \text { H24 } & 5.13042504 & -1.57869788 & 1.7990872 \\ \text { H25 } & 5.12389022 & -0.10962947 & 0.79836993 \\ \text { H26 } & 6.49296822 & -1.23993088 & 0.70665993 \\ \text { H27 } & 4.49049861 & -3.92872039 & -1.11076036 \\ \text { H28 } & 4.73541424 & -3.87997514 & 0.6462467 \\ \text { H29 } & 6.10270125 & -3.57206409 & -0.4500316\end{array}$




$\begin{array}{lrrr}\text { C30 } & -0.99730091 & 0.4447263 & 1.04955875 \\ \text { C31 } & -0.29514851 & 0.26688508 & 2.27005609 \\ \text { C32 } & -2.47881534 & 0.33591895 & 0.99938798 \\ \text { C33 } & 1.09337993 & 0.40459521 & 2.2280971 \\ \text { C34 } & -3.19637097 & -0.56823203 & 1.80248469 \\ \text { C35 } & -3.20045038 & 1.17474455 & 0.1329918 \\ \text { O36 } & 1.64659951 & 0.71020773 & 1.11259322 \\ \text { C37 } & 1.97856898 & 0.07260468 & 3.40889582 \\ \text { C38 } & -4.59438598 & 1.12961418 & 0.0832212 \\ \text { C39 } & -4.5891025 & -0.61748776 & 1.75230575 \\ \text { C40 } & -5.29400556 & 0.23282529 & 0.89436399 \\ \text { H41 } & -0.60058782 & 1.24170274 & 0.42640976 \\ \text { H42 } & -0.78820971 & -0.12156352 & 3.15548053 \\ \text { H43 } & -2.65791069 & -1.23935564 & 2.46649585 \\ \text { H44 } & -2.65830685 & 1.87625934 & -0.49748309 \\ \text { H45 } & 2.70820006 & 0.87343343 & 3.57234292 \\ \text { H46 } & 1.40026094 & -0.07430876 & 4.32675549 \\ \text { H47 } & 2.5433298 & -0.84382852 & 3.19623502 \\ \text { H48 } & -5.1327386 & 1.7943689 & -0.58743599 \\ \text { H49 } & -5.12641074 & -1.32164489 & 2.38234955 \\ \text { H50 } & -6.37948156 & 0.19379274 & 0.85684442\end{array}$

B3LYP/6-31G* Energy = -990.3744042

Number of Imaginary Frequencies $=1(-483.63)$

DATS 1a+2a-B

B3LYP/lacvp* Geometry

$\begin{array}{lrrr}\text { C1 } & 1.44569786 & -1.27911367 & -1.9528021 \\ \text { C2 } & 1.51388083 & 0.09310844 & -2.01520221 \\ \text { O3 } & 0.25192706 & -1.82703445 & -1.85586971 \\ \text { C4 } & 2.64301909 & -2.18579242 & -1.9225971 \\ \text { C5 } & 0.29539271 & 0.85600179 & -1.90665267 \\ \text { C6 } & 0.35481072 & 2.33985587 & -2.02361422 \\ \text { C7 } & 1.42633025 & 3.09139213 & -1.50913396 \\ \text { C8 } & -0.69173167 & 3.01985198 & -2.66904813 \\ \text { C9 } & 1.45605306 & 4.47809156 & -1.65081085 \\ \text { C10 } & -0.66119539 & 4.40731117 & -2.81600507 \\ \text { C11 } & 0.4132887 & 5.14078739 & -2.30654734 \\ \text { H12 } & 2.47750599 & 0.59025064 & -2.02620568 \\ \text { H13 } & 2.59146623 & -2.90034617 & -2.75315527 \\ \text { H14 } & 3.57969793 & -1.62499962 & -1.99533822 \\ \text { H15 } & 2.62300326 & -2.76525926 & -0.99391265 \\ \text { H16 } & -0.53881312 & 0.42164543 & -2.45133644 \\ \text { H17 } & 2.23589513 & 2.58707046 & -0.98821482 \\ \text { H18 } & -1.5300572 & 2.45055197 & -3.06408939 \\ \text { H19 } & 2.29177842 & 5.04354083 & -1.24707192 \\ \text { H20 } & -1.47614092 & 4.91437699 & -3.32558654 \\ \text { H21 } & 0.43738655 & 6.22178401 & -2.41567464 \\ \text { B22 } & -0.45842946 & -2.31661602 & -0.45943467 \\ \text { O23 } & -1.64666416 & -2.99173193 & -0.77588889 \\ \text { C24 } & -0.52143932 & 0.47541426 & -0.1808342 \\ \text { C25 } & -0.69520116 & -0.77400682 & -0.12344308 \\ \text { O26 } & 0.54193016 & -2.96356205 & 0.32653304 \\ \text { C27 } & -1.63192721 & -4.37441497 & -1.12552412 \\ \text { C28 } & 0.55857559 & -2.8059435 & 1.7405909 \\ \text { C29 } & -0.78345552 & 1.68743474 & 0.64789435 \\ \text { C30 } & -2.91050308 & -5.00454716 & -0.57326425\end{array}$

$\begin{array}{lrrr}\text { C31 } & -1.53054084 & -4.52922074 & -2.64651202 \\ \text { C32 } & -0.53150762 & -3.64432381 & 2.41726504 \\ \text { C33 } & 1.95535948 & -3.1807114 & 2.23277538 \\ \text { H34 } & -0.76164293 & -4.85523698 & -0.65733517 \\ \text { H35 } & 0.37673087 & -1.74421852 & 1.98272112 \\ \text { H36 } & 0.14804315 & 2.21947639 & 0.86873351 \\ \text { H37 } & -1.25779719 & 1.40506812 & 1.59193861 \\ \text { H38 } & -1.43980054 & 2.38855361 & 0.12127241 \\ \text { H39 } & -2.96746337 & -6.06933666 & -0.82908765 \\ \text { H40 } & -2.94979664 & -4.90700357 & 0.51683907 \\ \text { H41 } & -3.78894787 & -4.49887256 & -0.99160914 \\ \text { H42 } & -1.53059564 & -5.5882342 & -2.93365147 \\ \text { H43 } & -2.38183424 & -4.03700833 & -3.1319178 \\ \text { H44 } & -0.61288225 & -4.06489205 & -3.01912674 \\ \text { H45 } & -0.51194517 & -3.50306325 & 3.5051146 \\ \text { H46 } & -1.52226338 & -3.3612239 & 2.04847185 \\ \text { H47 } & -0.37853214 & -4.7089276 & 2.20366284 \\ \text { H48 } & 2.03376918 & -3.06291045 & 3.31986146 \\ \text { H49 } & 2.17636066 & -4.22456495 & 1.98028819 \\ \text { H50 } & 2.71220772 & -2.54598443 & 1.75919611\end{array}$

B3LYP/6-31G* Energy = -990.364823

Number of Imaginary Frequencies $=1(-391.58)$

Hetero Diels-Alder Reaction between 1a and 5a DATS 1a+5a-B-Re

B3LYP/lacvp* Geometry

$\begin{array}{lrrr}\text { C1 } & 2.99903076 & -0.0129946 & -0.69263237 \\ \text { C2 } & 2.93948492 & 1.33862899 & -0.52887355 \\ \text { O3 } & 1.87642985 & -0.72059559 & -0.83818986 \\ \text { C4 } & 4.27661406 & -0.79597441 & -0.75068356 \\ \text { C5 } & 1.63688249 & 2.00839125 & -0.45767097 \\ \text { B6 } & 0.79638346 & -1.12016834 & 0.11818823 \\ \text { C7 } & 1.59528718 & 3.50993386 & -0.48256875 \\ \text { O8 } & -0.30903005 & -1.52937593 & -0.71137989 \\ \text { C9 } & 0.85873191 & 1.51026133 & 1.07982083 \\ \text { C10 } & 0.56595997 & 0.29925175 & 0.85489396 \\ \text { O11 } & 1.24089844 & -2.08120357 & 1.10998849 \\ \text { C12 } & 2.60773865 & 4.30619702 & 0.07837195 \\ \text { C13 } & 0.50412117 & 4.14263401 & -1.10114313 \\ \text { C14 } & -1.50649461 & -1.66250259 & -0.1026736 \\ \text { C15 } & 0.73786561 & -3.3082339 & 1.33635529 \\ \text { C16 } & 0.75637664 & 2.51169814 & 2.18033562 \\ \text { C17 } & 2.5391885 & 5.69762391 & 0.00355669 \\ \text { C18 } & 0.43478215 & 5.53413053 & -1.17597078 \\ \text { C19 } & -1.69947342 & -2.64239265 & 0.89874058 \\ \text { C20 } & -2.58875641 & -0.8502236 & -0.48089918 \\ \text { C21 } & 1.61890949 & -4.31147066 & 1.78965525 \\ \text { C22 } & -0.64247546 & -3.63355483 & 1.23308799 \\ \text { C23 } & 1.45378998 & 6.31576629 & -0.62479275 \\ \text { C24 } & -2.95294561 & -2.71762461 & 1.53084411 \\ \text { C25 } & -3.83490709 & -0.95802177 & 0.13209962 \\ \text { I26 } & -2.32641665 & 0.57156036 & -2.04942729 \\ \text { C27 } & 1.19468453 & -5.60628782 & 2.07132153 \\ \text { C28 } & -1.04628576 & -4.95435536 & 1.49908884 \\ \text { I29 } & 3.67966908 & -3.85254631 & 2.10009908 \\ \text { C30 } & -4.00862508 & -1.89004959 & 1.15763891\end{array}$




$\begin{array}{rrrr}\text { C31 } & -0.15097376 & -5.93585454 & 1.9074607 \\ \text { H32 } & 3.85506758 & 1.89902237 & -0.38289972 \\ \text { H33 } & 4.32638563 & -1.35872921 & -1.69029888 \\ \text { H34 } & 5.15045188 & -0.14170383 & -0.68101641 \\ \text { H35 } & 4.30317488 & -1.52918644 & 0.06393027 \\ \text { H36 } & 0.93419361 & 1.60462476 & -1.18517728 \\ \text { H37 } & 3.45575386 & 3.83987803 & 0.57213147 \\ \text { H38 } & -0.28995851 & 3.5332613 & -1.5267643 \\ \text { H39 } & 1.74802452 & 2.89756199 & 2.43843088 \\ \text { H40 } & 0.31510972 & 2.04990139 & 3.06683715 \\ \text { H41 } & 0.13924396 & 3.36154196 & 1.87190124 \\ \text { H42 } & 3.33406481 & 6.29977908 & 0.43551915 \\ \text { H43 } & -0.41232052 & 6.0064378 & -1.66593129 \\ \text { H44 } & 1.40289405 & 7.39974362 & -0.68320772 \\ \text { H45 } & -3.0913868 & -3.4352334 & 2.33372136 \\ \text { H46 } & -4.65373096 & -0.31679088 & -0.17639586 \\ \text { H47 } & 1.90916251 & -6.35081399 & 2.40590456 \\ \text { H48 } & -2.0929227 & -5.20872751 & 1.36358695 \\ \text { H49 } & -4.96813782 & -1.96623437 & 1.66138552 \\ \text { H50 } & -0.4920831 & -6.94921226 & 2.09789159\end{array}$

B3LYP/6-31G* Energy = -1236.992262

Number of Imaginary Frequencies $=1(-179.33)$

Hetero Diels-Alder Reaction between 1a and 5a DATS 1a+5a-B-Si

B3LYP/lacvp* Geometry

$\begin{array}{lrrr}\text { C1 } & 1.13765268 & -0.37675585 & 2.28806929 \\ \text { C2 } & 1.18189982 & 0.98701776 & 2.36423313 \\ \text { O3 } & -0.06171602 & -0.95464837 & 2.24538692 \\ \text { C4 } & 2.33771003 & -1.27075753 & 2.194859 \\ \text { C5 } & -0.05934 & 1.73980934 & 2.31246664 \\ \text { B6 } & -0.84988425 & -1.33341832 & 0.98896884 \\ \text { C7 } & -0.01418045 & 3.22183771 & 2.48614754 \\ \text { O8 } & 0.0199604 & -2.07032974 & 0.09661437 \\ \text { O9 } & -2.07185818 & -1.98006066 & 1.3483579 \\ \text { C10 } & -0.86218516 & 1.43382006 & 0.61874274 \\ \text { C11 } & -1.092102 & 0.1923126 & 0.57494286 \\ \text { C12 } & 1.05457209 & 4.00430791 & 2.01359562 \\ \text { C13 } & -1.07353614 & 3.8633198 & 3.150285 \\ \text { C14 } & -0.4906817 & -2.79222611 & -0.92872356 \\ \text { C15 } & -2.09273063 & -3.29952007 & 1.65214989 \\ \text { C16 } & -1.02407738 & 2.63974322 & -0.24340668 \\ \text { C17 } & 1.06925128 & 5.38408743 & 2.21426248 \\ \text { C18 } & -1.05834705 & 5.24366794 & 3.35487609 \\ \text { C19 } & -1.35874089 & -3.88625804 & -0.69264056 \\ \text { C20 } & -0.10070391 & -2.51188655 & -2.24795585 \\ \text { C21 } & -2.54031383 & -3.71995961 & 2.91405312 \\ \text { C22 } & -1.75247447 & -4.27537212 & 0.68541693 \\ \text { C23 } & 0.01360932 & 6.0082658 & 2.88675748 \\ \text { C24 } & -1.81959856 & -4.63226543 & -1.78847092 \\ \text { C25 } & -0.55353512 & -3.27091174 & -3.32567642 \\ \text { I26 } & 1.23140408 & -0.88795847 & -2.62711954 \\ \text { C27 } & -2.63487478 & -5.07160095 & 3.24063883 \\ \text { C28 } & -1.83846732 & -5.63232478 & 1.03326944 \\ \text { I29 } & -3.1061741 & -2.26612788 & 4.36596531 \\ \text { C30 } & -1.42478601 & -4.33548844 & -3.09078938 \\ & & & \end{array}$

$\begin{array}{lrrr}\text { C31 } & -2.27358889 & -6.03213752 & 2.29420275 \\ \text { H32 } & 2.13766916 & 1.49791173 & 2.35572781 \\ \text { H33 } & 3.26697636 & -0.70533013 & 2.30966656 \\ \text { H34 } & 2.33627668 & -1.77717886 & 1.22296376 \\ \text { H35 } & 2.28982413 & -2.04620928 & 2.96789649 \\ \text { H36 } & -0.85755243 & 1.28333634 & 2.89239586 \\ \text { H37 } & 1.87653746 & 3.53281901 & 1.4819001 \\ \text { H38 } & -1.90949884 & 3.27030391 & 3.51388716 \\ \text { H39 } & -0.05358088 & 3.1104207 & -0.43201952 \\ \text { H40 } & -1.46835018 & 2.35745328 & -1.20113618 \\ \text { H41 } & -1.66253188 & 3.38515592 & 0.24205252 \\ \text { H42 } & 1.90374048 & 5.97377797 & 1.84425739 \\ \text { H43 } & -1.88248462 & 5.72114467 & 3.87788775 \\ \text { H44 } & 0.02627728 & 7.0838215 & 3.04207952 \\ \text { H45 } & -2.51171807 & -5.4493318 & -1.60774289 \\ \text { H46 } & -0.23715985 & -3.02811615 & -4.33453065 \\ \text { H47 } & -2.97925556 & -5.37003011 & 4.22512362 \\ \text { H48 } & -1.54433857 & -6.3786733 & 0.30108925 \\ \text { H49 } & -1.79699777 & -4.92368653 & -3.92474814 \\ \text { H50 } & -2.32899229 & -7.08731071 & 2.54625908\end{array}$

B3LYP/6-31G* Energy = -1236.995781

Number of Imaginary Frequencies $=1(-331.46)$ 

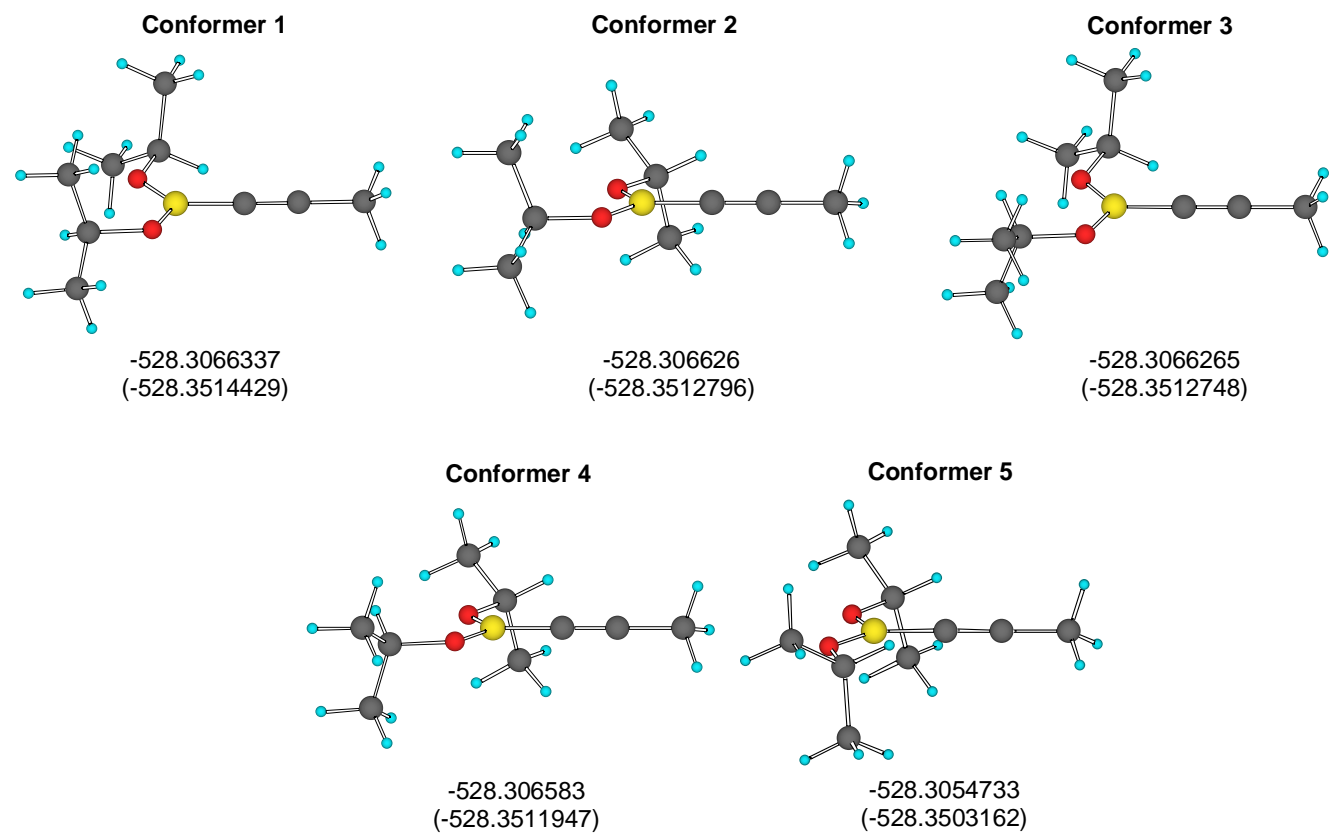

Figure S4. B3LYP/lacvp* geometries and absolute energies (in hartrees), including ZPE (free energies in parentheses) of the conformers located for alkynylboronate $\mathbf{2 a}$.
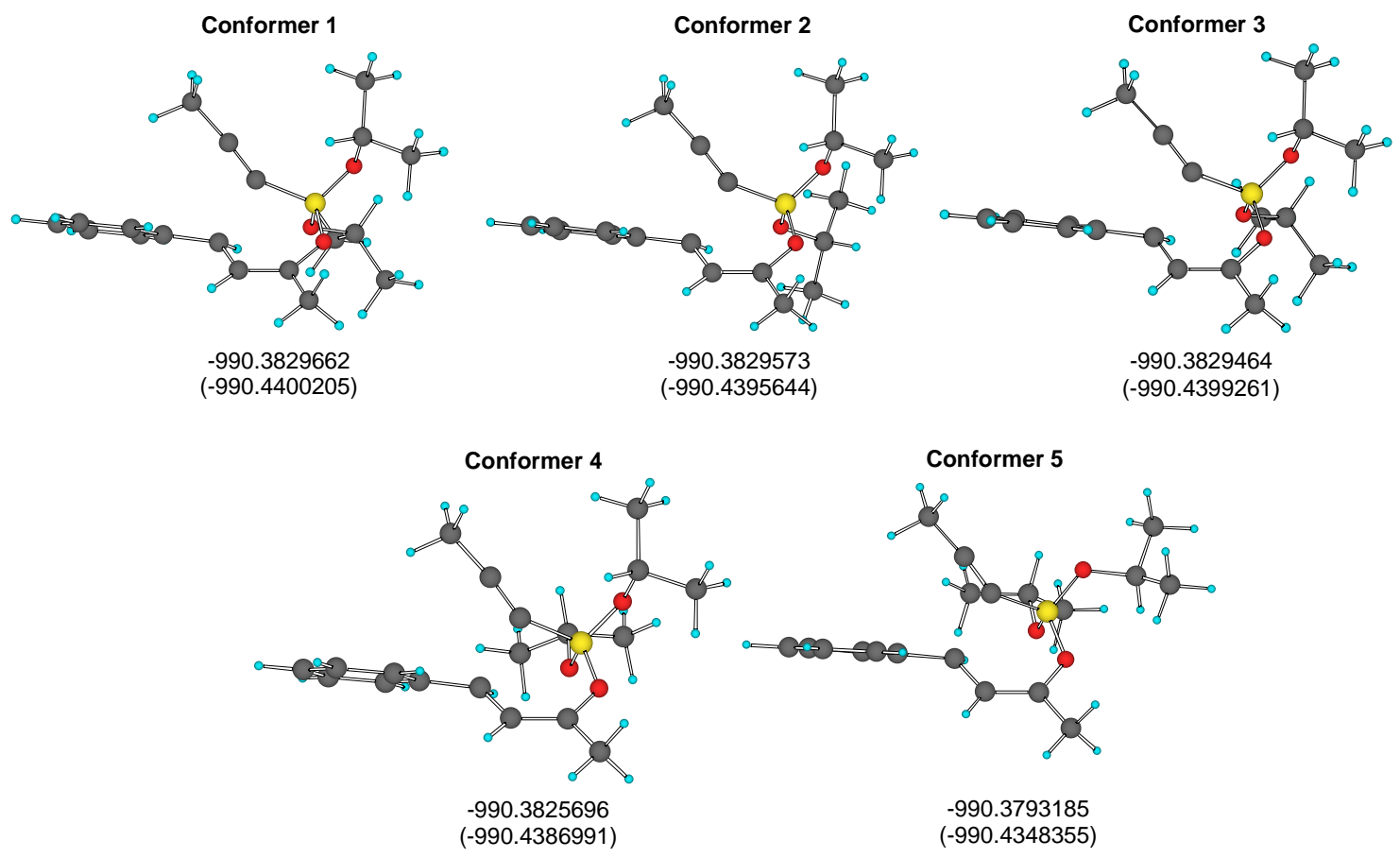

Figure S5. B3LYP/lacvp* geometries and absolute energies (in hartrees), including ZPE (free energies in parentheses) of the conformers located for the transition structure of the conjugate addition of alkynylboronate $\mathbf{2 a}$ to enone $1 \mathbf{a}$ (TS $1 \mathbf{a}+2 \mathbf{a})$. 

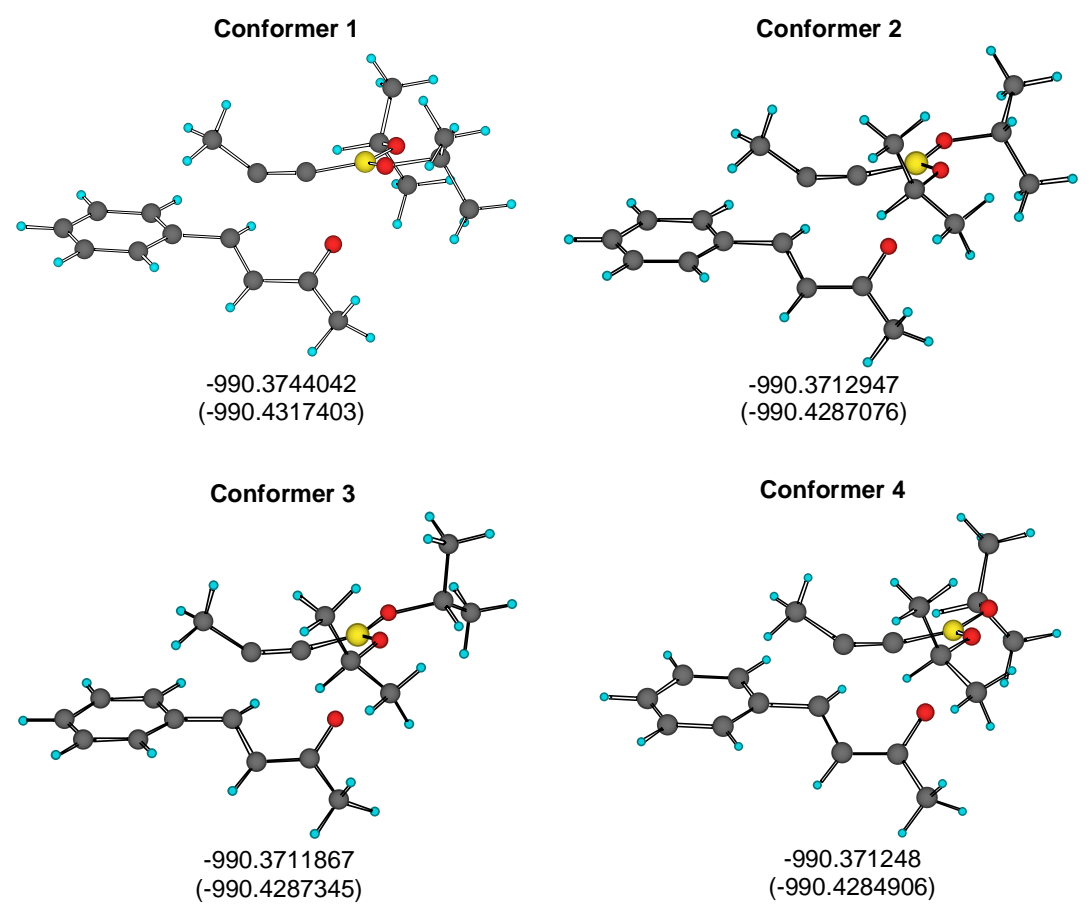

Figure S6. B3LYP/lacvp* geometries and absolute energies (in hartrees), including ZPE (free energies in parentheses) of the conformers located for the transition structure of hetero Diels-Alder reaction of enone 1a with alkynylboronate 2a (DATS $1 \mathbf{a}+\mathbf{2 a}-\mathbf{A})$.

Table S1. Energies and Atomic Coefficients of the Frontier Molecular Orbitals of the Reactants at the B3LYP/lacvp* Level.

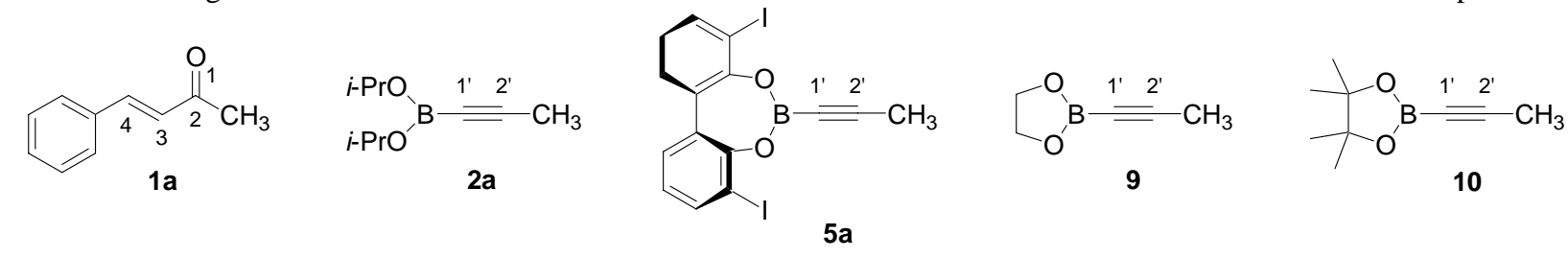

\begin{tabular}{|c|c|c|c|c|c|c|c|c|c|c|c|c|c|c|c|}
\hline & & \multicolumn{2}{|c|}{$\mathrm{O}_{1}$} & \multicolumn{2}{|c|}{$\mathrm{C}_{2}$} & \multicolumn{2}{|c|}{$\mathrm{C}_{3}$} & \multicolumn{2}{|c|}{$\mathrm{C}_{4}$} & \multicolumn{2}{|c|}{$\mathrm{B}$} & \multicolumn{2}{|c|}{$\mathrm{C}_{1}^{\prime}$} & \multicolumn{2}{|c|}{$\mathrm{C}_{2}^{\prime}$} \\
\hline & $\mathrm{E}(\mathrm{eV})$ & $2 \mathrm{pz}$ & $2 \mathrm{pz}^{\prime}$ & $2 \mathrm{pz}$ & $2 \mathrm{pz}^{\prime}$ & $2 \mathrm{pz}$ & $2 \mathrm{pz}^{\prime}$ & $2 \mathrm{pz}$ & $2 \mathrm{pz}^{\prime}$ & $2 p z$ & $2 \mathrm{pz}^{\prime}$ & $2 \mathrm{pz}$ & $2 \mathrm{pz}^{\prime}$ & $2 \mathrm{pz}$ & $2 \mathrm{pz}^{\prime}$ \\
\hline HOMO 1a & -0.23 & 0.20 & 0.15 & -0.01 & -0.01 & -0.31 & -0.25 & -0.19 & -0.12 & & & & & & \\
\hline LUMO 1a & -0.07 & -0.25 & -0.24 & 0.24 & 0.20 & 0.23 & 0.29 & -0.30 & -0.34 & & & & & & \\
\hline HOMO 2a & -0.25 & & & & & & & & & 0.00 & 0.04 & 0.40 & 0.22 & 0.39 & 0.26 \\
\hline LUMO 2a & 0.01 & & & & & & & & & -0.35 & -0.34 & -0.21 & -0.29 & 0.37 & 0.41 \\
\hline HOMO $5 a^{a}$ & -0.23 & & & & & & & & & - & - & - & - & - & - \\
\hline LUMO 5a & -0.05 & & & & & & & & & -0.19 & -0.17 & -0.04 & -0.05 & 0.14 & 0.13 \\
\hline HOMO 9 & -0.26 & & & & & & & & & -0.01 & -0.06 & -0.41 & -0.42 & -0.39 & -0.26 \\
\hline LUMO 9 & 0.00 & & & & & & & & & 0.36 & 0.36 & 0.20 & 0.27 & -0.37 & -0.41 \\
\hline HOMO 10 & -0.25 & & & & & & & & & 0.00 & -0.06 & -0.39 & -0.21 & -0.38 & -0.25 \\
\hline LUMO 10 & 0.00 & & & & & & & & & 0.36 & 0.35 & 0.20 & 0.27 & -0.37 & -0.41 \\
\hline
\end{tabular}

${ }^{\mathrm{a}}$ This $\mathrm{MO}$ is localized on the oxygens and the carbons of the biphenyl moiety. However, there are two occupied orbitals $(\mathrm{E}=$ -0.27 and $-0.26 \mathrm{ev}$ ) that are associated with $\mathrm{C}_{1}$ ' and $\mathrm{C}_{2}$ '. This makes the $\mathrm{HOMO}_{\text {enone }}-\mathrm{LUMO}_{\text {alkynylboronate }}$ the most favorable interaction for 1a and 5a which leads to the formation of the complex between the reactants and facilitates the subsequent conjugate addition of the alkyne to the enone system by lowering the HOMO-LUMO energy gap to $0.12 \mathrm{ev}$. 